\title{
Psychological Responses to Acute Exercise in Sedentary Black and White Individuals
}

\author{
Rebecca E. Hasson, Kirsten E. Granados, David Xavier Marquez, Gary Bennett, \\ Patty Freedson, and Barry Braun
}

\begin{abstract}
Background: Racial differences in psychological determinants of exercise exist between non-Hispanic blacks (blacks) and non-Hispanic whites (whites). To date, no study has examined racial differences in the psychological responses during and after exercise. The objective of this study was to compare psychological outcomes of single exercise bouts in blacks and whites. Methods: On 3 separate occasions, sedentary black $(\mathrm{n}=16)$ and white $(\mathrm{n}=14)$ participants walked on a treadmill at $75 \%$ max HR for 75 minutes. Questionnaires assessing mood, state anxiety, and exercise task self-efficacy were administered before and after each exercise bout. In-task mood and rating of perceived exertion (RPE) were measured every 5 minutes during exercise. Results: Exercise self-efficacy and psychological distress significantly improved in both blacks and whites. However during exercise blacks reported more positive in-task mood and lower RPE compared with whites. Conclusions: These data suggest that racial differences exist in psychological responses during exercise. Further research should confirm these findings in a larger, free-living population.
\end{abstract}

Keywords: race, self-efficacy, mood, state anxiety

Despite the benefits of leisure time physical activity (LTPA), more than half of adults in the United States are not regularly active at the recommended levels, with racial minority populations reporting the highest prevalence of no LTPA. ${ }^{1}$ Specifically, rates of physical inactivity are significantly higher in non-Hispanic blacks (blacks; men $=27.0 \%$, women $=33.9 \%$ ) compared with non-Hispanic whites $($ whites; men $=18.4 \%$, women $=21.6 \%){ }^{2}$

Social Cognitive Theory (SCT) ${ }^{3}$ has been used extensively to explain and influence exercise behavior. SCT describes human behavior as resulting from the dynamic interactions among cognition, behavior and environment, which operates interactively to influence current and future behavior. ${ }^{3}$ This dynamic relationship is characterized as triadic reciprocal causation. Constructs emerging from SCT, including exercise self-efficacy, social support for exercise, outcome expectations and perceived barriers, have repeatedly been identified as important predictors of exercise behavior in the general population. ${ }^{3}$ For example, self-regulatory exercise self-efficacy (confidence in one's ability to exercise on a regular basis) and social support for exercise (tasks or steps that significant others take to facilitate an exercise behavior) have been positively associated with LTPA. ${ }^{3}$ Additionally, individuals who

Hasson, Granados, Freedson, and Braun are with the Dept of Kinesiology, University of Massachusetts, Amherst, MA. Marquez is with the Depts of Kinesiology and Nutrition, University of Illinois at Chicago. Bennett is with the Dept of Psychology, Duke University, Durham, NC. report changes in these variables (eg, postintervention improvements in self-regulatory exercise self-efficacy and outcome expectations) are also predicted to increase their exercise behavior. ${ }^{3}$

Self-efficacy is one of the most widely studied psychosocial correlates of physical activity for the general population. Despite lower LTPA rates in racial minorities, several studies have reported greater than or equal self-efficacy scores in blacks as compared with whites. ${ }^{4-8}$ In addition, blacks tend to report higher levels of social support for exercise compared with whites ${ }^{5,9}$ and cite similar outcome expectations for exercise compared with whites. ${ }^{10}$ Nevertheless, improvements in self-efficacy and social support for exercise increase exercise behavior in both races. ${ }^{3.11}$

The psychological benefits of regular physical activity engagement are also well documented..$^{2.12}$ Multiple reviews and meta-analyses have reported that exercise has a beneficial effect on depression, ${ }^{13,14}$ anxiety, ${ }^{14,15}$ and cognitive functioning (eg, executive control processes). ${ }^{13}$ Furthermore, single bouts of exercise are associated with improved mood, ${ }^{16,17}$ decreased state-anxiety, ${ }^{15}$ and improved exercise task self-efficacy. ${ }^{18}$ Only recently has there been systematic investigation into the responses occurring during exercise. ${ }^{19}$ However, few studies have examined the psychological response before, during and after exercise in racial minorities.

To date, only 1 study has investigated ethnic/racial differences in anxiety responses to a single bout of exercise. Marquez et $\mathrm{al}^{20}$ manipulated exercise task selfefficacy and examined its effect on the state anxiety of 
low active women. The sample consisted primarily of Latina women $(n=31)$ and non-Latina whites $(n=24)$. Participants were randomly assigned to a low or high efficacy condition, and self-efficacy was manipulated by presentation of computer-generated false feedback after a graded exercise test. Efficacy was successfully manipulated and participants randomized to the high efficacy condition reported significantly less anxiety than those in the low efficacy condition both after the graded exercise test and before and after a single bout of exercise. Interestingly, Latina women were less anxious at all time points of the exercise bout. To our knowledge, no study has directly examined potential racial differences in psychological outcomes to single exercise bouts in blacks and whites. Therefore, the purpose of this study was to compare the psychological responses to single bouts of exercise in age/sex/BMI-matched blacks and whites. It was hypothesized that after each bout of exercise blacks would have a smaller improvement in exercise task selfefficacy, state-anxiety, and mood compared with whites. Furthermore, it was hypothesized that black participants would have lower affect and rating of perceived exertion (RPE) during exercise compared with white participants.

\section{Methods}

\section{Participants}

As previously stated SCT describes the bidirectional influence of behavior and environment on cognition. ${ }^{3}$ Therefore, to minimize differences in social/environmental determinants of physical activity (eg, education level, neighborhood safety, lack of transportation, lack of access to gyms) as well as behavior (eg, LTPA) 30 sedentary but otherwise healthy men and women from the university community were recruited to participate in this study. Sedentary was defined as doing less than 60 minutes of moderate to vigorous activity per week as assessed by Physical Activity Rating Questionnaire (PAS). All participants belonged to one of two racial groups: nonHispanic black or non-Hispanic white. Race was defined as persons having resided in the United States for the past
5 years who self-identified themselves as belonging to either the black or white race. All participants were free from cardiovascular, respiratory, metabolic and mental health disorders (ie, hypertension, asthma, coronary heart disease, Type 2 diabetes, or depression). All participants read and signed an informed consent document approved by the University Institutional Review Board.

\section{Measures}

To assess prepost changes in psychological outcomes of exercise, questionnaires assessing mood, exercise task self-efficacy, and state anxiety were administered before and after each bout of exercise. The timetable for psychological measurements is presented in Table 1.

The Exercise Self-Efficacy for Duration Scale ${ }^{21}$ assessed the participants' belief in their ability to walk at a moderate intensity without stopping. The scale consisted of 6 items, with each item representing 1 10-minute increment. The responses were scored on a 100-point percentage scale $(100 \%=$ complete confidence, $0 \%=$ no confidence at all). Summing the efficacy scores and dividing by the total number of items in the scale calculated an average efficacy score, resulting in a maximum possible efficacy score of 100 . This scale has been shown to be valid and reliable in the general population, including racial minorities. ${ }^{19-21}$

The State Trait Anxiety Inventory (STAI) ${ }^{22}$ is the definitive instrument for measuring anxiety in adults. The STAI has 20 questions with 4 possible responses (1 $=$ not at all, $4=$ very much so). The items were summed to produce a total score in which higher scores equate to greater state anxiety. The STAI has been shown to be valid and reliable. ${ }^{7}$

To evaluate pre- and post- exercise changes in mood, participants were asked to complete the Subjective Exercise Experiences Scale (SEES). ${ }^{23}$ The SEES is a 12-item, 3-dimensional scale that assessed 3 general categories of mood responses to exercise: positive wellbeing (eg, great), psychological distress (eg, miserable), and fatigue (eg, tired). For each item participants were asked to indicate how strongly they were experiencing the

Table 1 Time Table for Psychological Measurements

\begin{tabular}{lccc}
\hline & \multicolumn{3}{c}{ Exercise bout 1-3 } \\
\cline { 2 - 4 } Measurement & Pre & During & Post \\
\hline Task self-efficacy & $\mathrm{x}$ & $\mathrm{x}$ \\
State anxiety & $\mathrm{x}$ & $\mathrm{x}$ \\
Mood & $\mathrm{x}$ & $\mathrm{x}$ \\
In-task mood & & $\mathrm{x}$ & \\
Rating of perceived exertion & & $\mathrm{x}$ & \\
Heart rate & & $\mathrm{x}$ & \\
\hline
\end{tabular}


feeling state at that time. Items were scored on a 7-point Likert scale ( 1 = "not at all," 7 = "very much so"). Each subscale ranged from 4 to 28 with higher scores representing greater fatigue, positive well-being or psychological distress. The SEES has been shown to have a high internal consistency across a variety of populations. ${ }^{23,24}$

To evaluate in-task mood responses during exercise, the Feeling Scale 25,26 was employed. Every 5 minutes during exercise participants were asked, "How do you feel right now?" Participants responded by selecting from an 11-point scale $(-5=$ "I feel very bad" through 0 $=$ neutral, to $+5=$ "I feel very good").

To determine perceived exercise intensity during exercise Rating of Perceived Exertion (RPE) ${ }^{27,28}$ was used. Participants were asked: "How hard do you feel you are working?" Participants responded by selecting from a 15 -point scale $(6=$ "no exertion at all" to $20=$ "maximal exertion"). Along with RPE scores, heart rate was taken every 5 minutes. The time course of changes in heart rate, RPE and in-task mood measures was examined across each bout of exercise.

\section{Procedures}

Participants walked on a treadmill at $75 \%$ of maximal heart rate for 75 minutes ( 4 periods of 15 minutes at a predetermined speed, interspersed with 5-minutes at a slow speed of $2.0 \mathrm{mph}$ ). Each exercise session included a 5-minute warm-up and cool-down period. Breath samples were taken during 1 15-minute bout using a Metabolic Cart (ParvoMedics TrueMax 2400, Consentius Technology, Sandy, UT) and a heart rate monitor was continuously worn to objectively verify exercise intensity. Psychological responses to exercise can be influenced by the context in which the exercise takes place. ${ }^{3}$ To minimize environmental influences on the data obtained, day of the week (weekends excluded), time of day (evening hours), and visual and auditory stimuli (television viewing during exercise) were standardized. In addition, participants were not provided with any feedback regarding their physiological state (ie, kilocalories burned, heart rate response during exercise) until after they had completed the study. To assess the cumulative change in psychological responses to exercise, this protocol was repeated at the same time of day and same day of the week (excluding weekends) for 3 consecutive weeks.

\section{Statistical Analysis}

Racial differences in participant characteristics (age, height, weight, BMI, exercise energy expenditure, oxygen consumption during exercise, and habitual physical activity) were assessed using 2-sample $t$ tests. Main effects and interactions of race, time, and exercise bout were assessed using a 3-way repeated measures analysis of covariance (ANCOVA), after controlling for sex. Analyses were performed for all main outcome variables to test for sphericity and Greenhouse-Gauser adjustments were made when necessary. When significant differences across race, time and exercise bout were identified, post hoc pairwise comparisons with Bonferroni adjustments were conducted. Effect size for all main outcome variables were calculated using partial Eta squared values. For all analyses, $P<.05$.

\section{Results}

\section{Participant Characteristics}

Participant characteristics are presented in Table 2. Sixteen blacks and 14 whites of similar age, BMI, and physical activity status completed the study protocol. No significant differences were detected between the 2 groups for any of the baseline characteristics or exercise intensity.

\section{Psychological Outcomes for Exercise}

Mean change in exercise task self-efficacy scores are presented in Figure 1. There was a significant effect of

Table 2 Subject Characteristics (Mean \pm SD)

\begin{tabular}{lccc}
\hline Participant characteristics & Black & White & $\boldsymbol{P}$ \\
\hline Gender (females/males) & $11 \mathrm{~F} / 5 \mathrm{M}$ & $10 \mathrm{~F} / 4 \mathrm{M}$ & - \\
Age (years) & $26.0 \pm 7.2$ & $22.3 \pm 6.9$ & 0.42 \\
Height (in) & $66.4 \pm 3.4$ & $66.9 \pm 3.5$ & 0.99 \\
Weight $(\mathrm{kg})$ & $81.6 \pm 9.5$ & $77.3 \pm 19.0$ & 0.50 \\
Body mass index $\left(\mathrm{kg} / \mathrm{m}^{2}\right)$ & $28.9 \pm 4.4$ & $26.6 \pm 5.5$ & 1.45 \\
Exercise energy expenditure $(\mathrm{kcals})$ & $501.7 \pm 107.8$ & $463.3 \pm 136.4$ & 0.37 \\
Exercise oxygen consumption $(\mathrm{L} / \mathrm{min})$ & $1.7 \pm 0.4$ & $1.6 \pm 0.5$ & 0.92 \\
Exercise oxygen consumption $(\mathrm{mL} / \mathrm{kg} / \mathrm{min})$ & $20.6 \pm 2.9$ & $19.0 \pm 4.8$ & 0.84 \\
Heart rate (bpm) & $137 \pm 2.5$ & $132 \pm 3.1$ & 0.20 \\
Physical activity score & $1.5 \pm 0.8$ & $1.8 \pm 0.6$ & 0.30 \\
\hline
\end{tabular}


exercise bout $\left(P<.01\right.$, partial $\left.\eta^{2}: 0.23\right)$ and a trend for time $\left(P=.08\right.$, partial $\left.\eta^{2}: 0.13\right)$ on self-efficacy scores. In addition, there was a significant time by exercise bout interaction $\left(P=.04\right.$, partial $\left.\eta^{2}: 0.15\right)$. Postexercise selfefficacy scores significantly improved in both black and white participants as a result of exercise bouts 1 and 2 .

Mean change in psychological distress scores are presented in Figure 2. There was a significant effect of race $\left(P<.01\right.$, partial $\left.\eta^{2}: 0.36\right)$ and time $(P<.01$, partial $\left.\eta^{2}: 0.35\right)$ on psychological distress scores. Blacks reported significantly lower levels of psychological distress compared with white participants, however, exercise significantly decreased psychological distress to a similar degree in both groups. Fatigue scores were also significantly lower in blacks compared with whites $\left(P<.01\right.$, partial $\left.\eta^{2}: 0.30\right)$, however there was no effect of

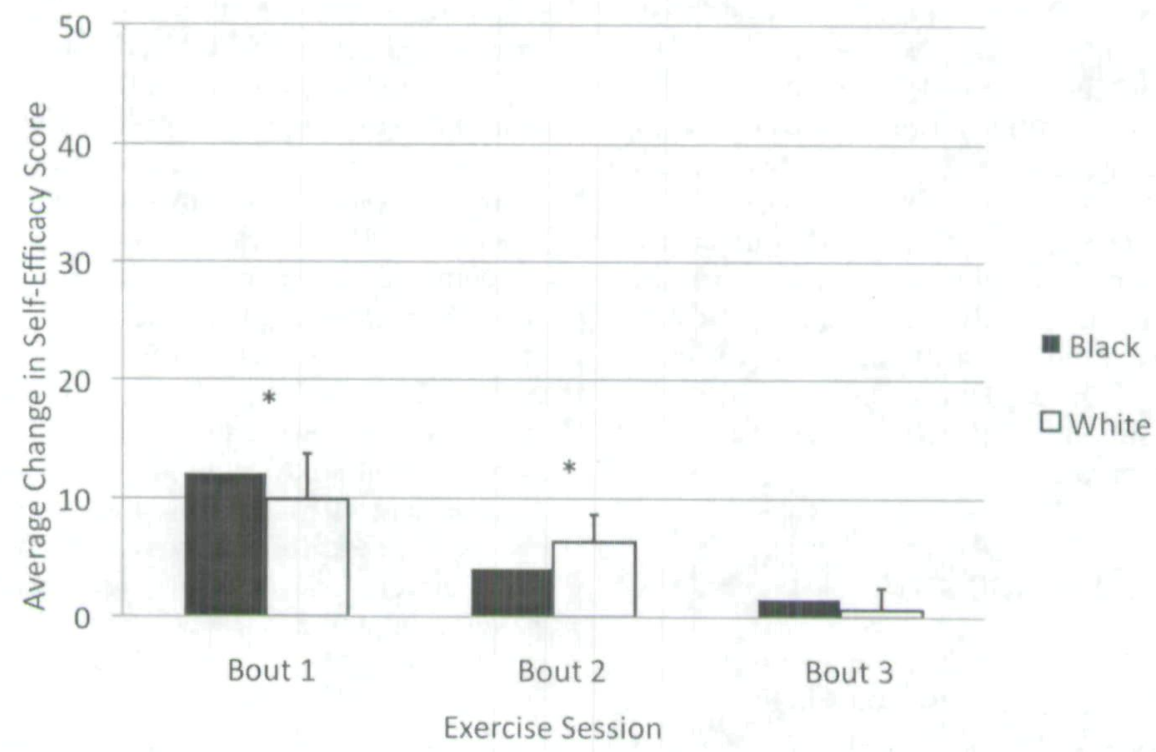

Figure 1 - Mean change in exercise task self-efficacy for duration scores. Mean \pm SE. * Significant effect of exercise at $P<.05$.

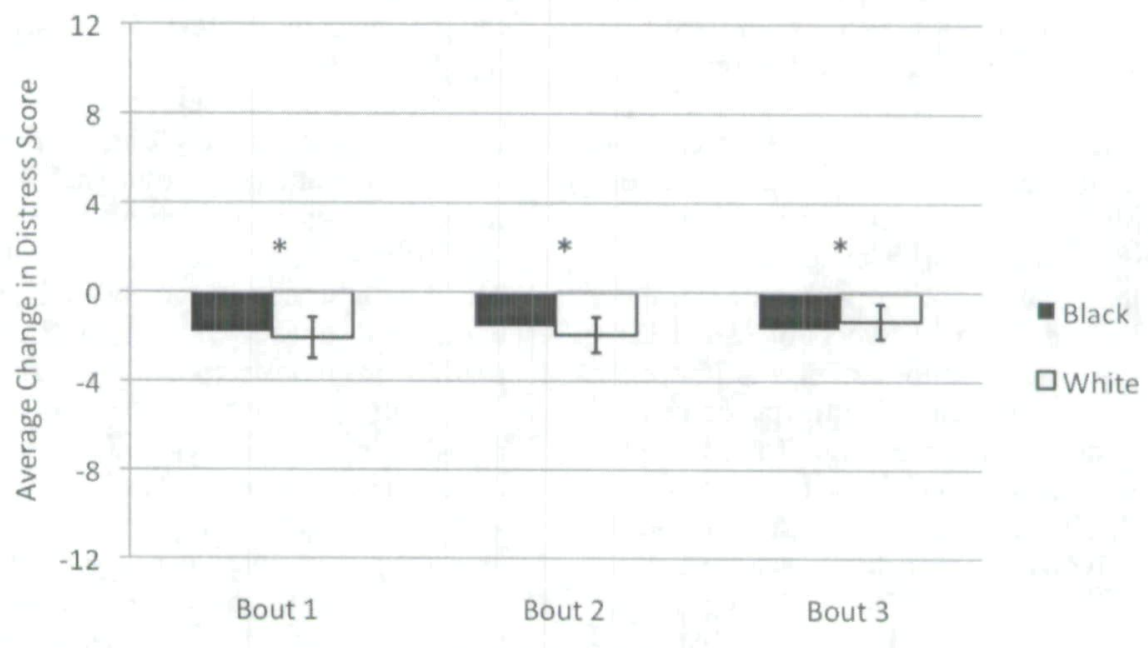

Exercise Session

Figure 2 - Mean change in mood (psychological distress) scores. Mean $\pm \mathrm{SE}$. * Significant effect of exercise at $P<.05$. 
exercise on fatigue scores in either group. Furthermore, there were no significant effects of exercise on measures of state-anxiety and positive well-being.

In-task mood, RPE and heart rate scores during exercise are presented in Figures 3 to 5 . For in-task mood, there was a significant effect of race $\left(P<.01\right.$, partial $\eta^{2}$ : $0.36)$ and time $\left(P<.01\right.$, partial $\left.\eta^{2}: 0.49\right)$. Both groups reported lower in-task mood scores during exercise compared with the beginning and end of exercise; however, black participants reported a more positive mood state $(P=.003)$ compared with white participants during all 3 exercise bouts. For RPE, there was a significant effect of race $\left(P=.02\right.$, partial $\left.\eta^{2}: 0.22\right)$, exercise bout $(P<.01$, partial $\left.\eta^{2}: 0.23\right)$, and time $\left(P<.01\right.$, partial $\left.\eta^{2}: 0.82\right)$. In addition, there was a significant time by exercise bout interaction $\left(P<.01\right.$, partial $\left.\eta^{2}: 0.11\right)$ and exercise bout by race interaction $\left(P=.04\right.$, partial $\left.\eta^{2}: 0.16\right)$. During exercise bout 3 only, black participants reported significantly lower RPE compared with white participants. For heart rate, there was a significant effect of duration $(P<.01$, partial $\left.\eta^{2}: 0.78\right)$, with heart rate increasing during exercise compared with the beginning and end of exercise. There were however no significant effects of race or exercise bout on heart rate scores.

\section{Discussion}

The primary aim of this study was to compare psychological outcomes of individual exercise bouts in blacks and age/sex/BMI-matched whites. The major findings of this study were that single bouts of exercise improved exercise task self-efficacy and reduced psychological distress in both black and white participants with no apparent racial differences in these psychological responses after exercise. Despite similar heart rates in both groups, racial differences were apparent during exercise with black participants reporting more positive in-task mood during all 3 bouts of exercise as well as lower RPE scores during the third bout.

It is recognized that individual bouts of exercise do not necessarily reflect the psychological adaptations seen with chronic (weeks/months) exercise training. However, a body of research has documented that many of the improvements in exercise task self-efficacy, stateanxiety, and mood are transient effects of recent exercise. ${ }^{16,18,29,30}$ Hence, each bout of exercise is important and supports our focus on optimizing the psychological effects garnered after each exercise bout. Enhancing the improvements associated with a single bout of exercise may maximize the efficacy of each exercise bout and the probability that an individual will engage in a subsequent exercise bout.

In response to exercise bouts 1 and 2, both black and white participants significantly increased exercise-task self-efficacy. During exercise bout 3, black and white participants reported high preexercise levels of exercise task self-efficacy $(83.9 \pm 8.0$ vs. $87.5 \pm 15.8$, respectively); therefore it is likely that participants maximized their potential to improve their exercise task self-efficacy by successfully completing 2 previous bouts of exercise at the same intensity and duration. The overall improvements in exercise task self-efficacy were similar to previous research demonstrating the beneficial effect of acute bouts of exercise on self-efficacy regardless of race. ${ }^{11}$

Previous research has demonstrated that single bouts of exercise are associated with improved $\operatorname{mood}^{25,26}$ and decreased state-anxiety. ${ }^{30}$ Petruzzello et $\mathrm{al}^{30}$ examined the effect of treadmill running at $75 \% \mathrm{VO}_{2} \max$ for short (15 minutes) and longer durations (30 minutes) on state anxiety and affect scores. Rudolph et $\mathrm{al}^{26}$ investigated the effect of 10-, 15-, and 20-minute bouts of treadmill running on affect scores at moderate intensity. All participants reported increased positive well-being and decreased psychological distress in all 3 conditions from baseline to 20 minutes after exercise. Finally, Daley et $\mathrm{al}^{25}$ compared the effect of 15 minutes versus a 30 minute bout of exercise on individuals' affect scores both during and after exercise. Positive affective responses were reported after both the 15 and 30-minute exercise bout and these effects were still evident 2 hours postexercise. In the current study, both black and white participants reported significantly lower levels of psychological distress after each bout of exercise, however no improvements in well-being or fatigue were observed. In addition, exercise did not reduce state-anxiety in either group. To ensure that our sedentary population could successfully complete each exercise session, we prescribed an exercise intensity and duration corresponding to current Institute of Medicine guidelines $\left(\sim 60 \% \mathrm{VO}_{2} \max\right.$ for 60 minutes including 3 to 5 minute active recovery sessions). ${ }^{31}$ It is possible that the exercise dose prescribed in the current study was sufficient to elicit improvements in psychological distress but the longer duration of exercise may have blunted the improvements in positive well-being, fatigue and state-anxiety previously reported at 15 - and 30 -minutes of exercise. If this prescription of exercise is used in future studies, researchers should also assess mood and state-anxiety during the 5-minute recovery periods that take place after each 15-minute interval of moderate intensity exercise.

To our knowledge, this is the first study to assess racial differences in psychological responses during exercise in blacks and whites. Both black and white participants reported positive in-task mood scores at the start (minute 1), during, and end of exercise (minute 75), however in-task mood declined significantly throughout the exercise session. In black participants mood scores were never negative and positive in-task mood was higher compared with white participants during all 3 bouts of exercise. In addition, RPE decreased progressively with each bout of exercise in blacks compared with whites, despite similar heart rates in both groups. These findings suggest that each bout of exercise became progressively easier for black participants and they felt better during exercise as well. It is unclear why black participants 
Bout 1

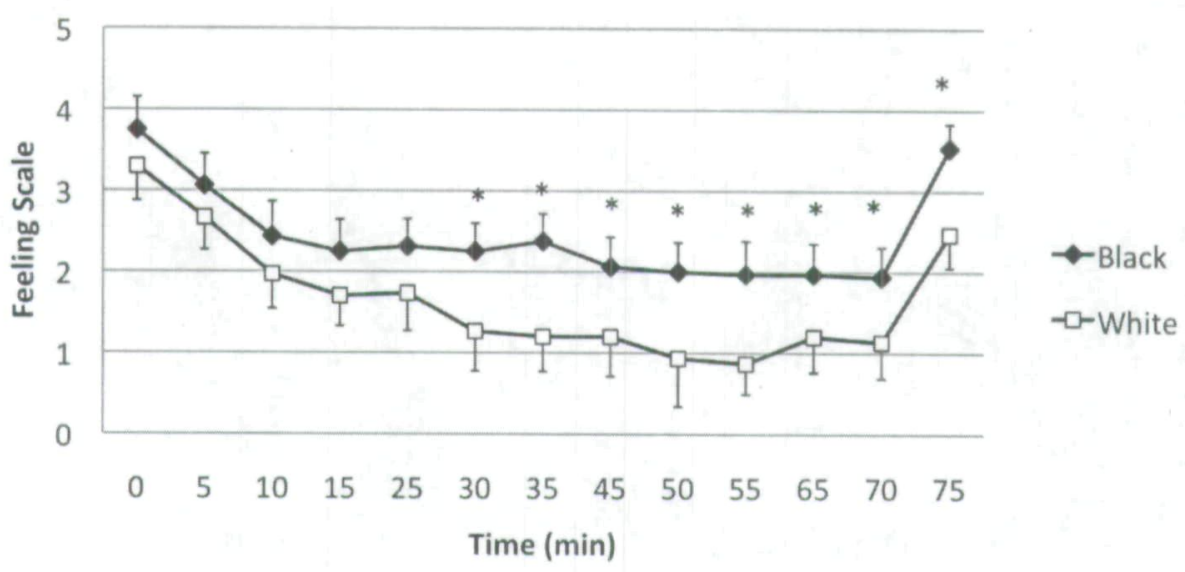

Bout 2

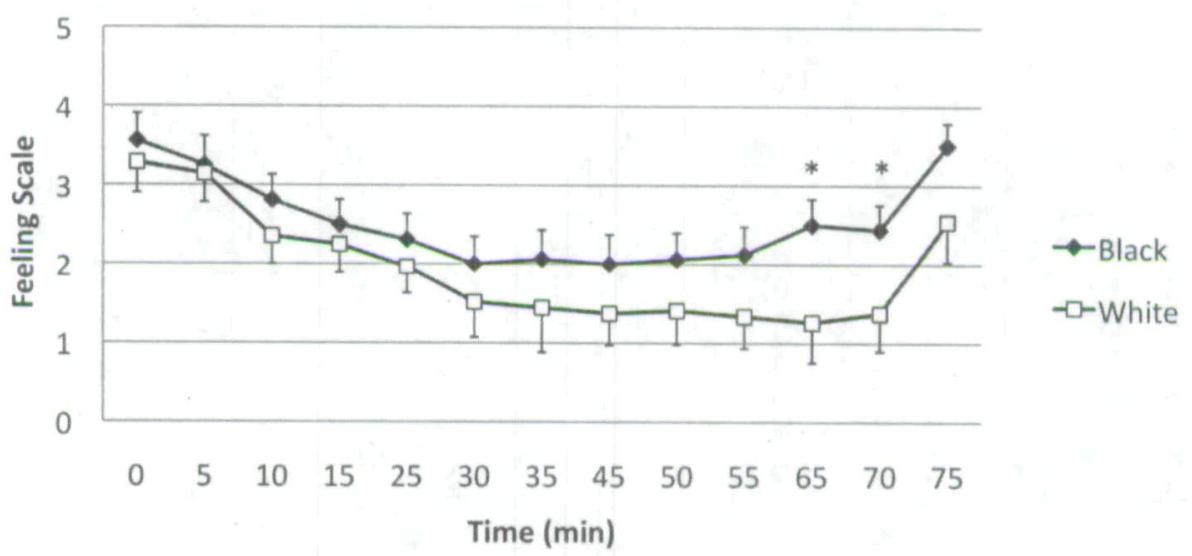

Bout 3

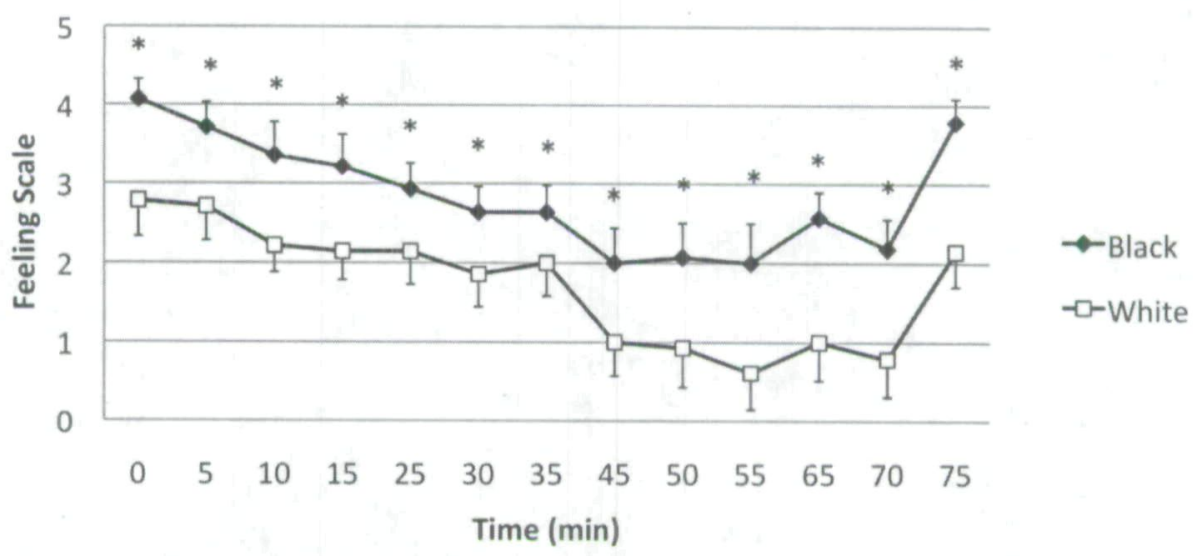

Figure 3 - In-task mood score during exercise for each exercise bout. Mean \pm SE. * Significant effect of exercise at $P<.05$. 
Bout 1

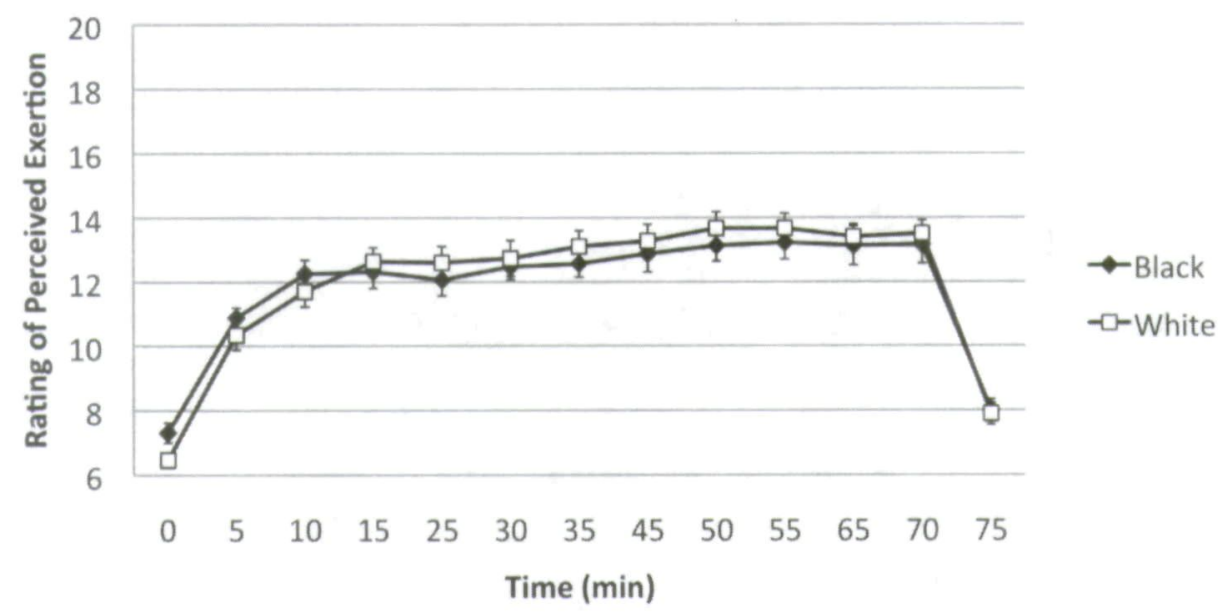

Bout 2

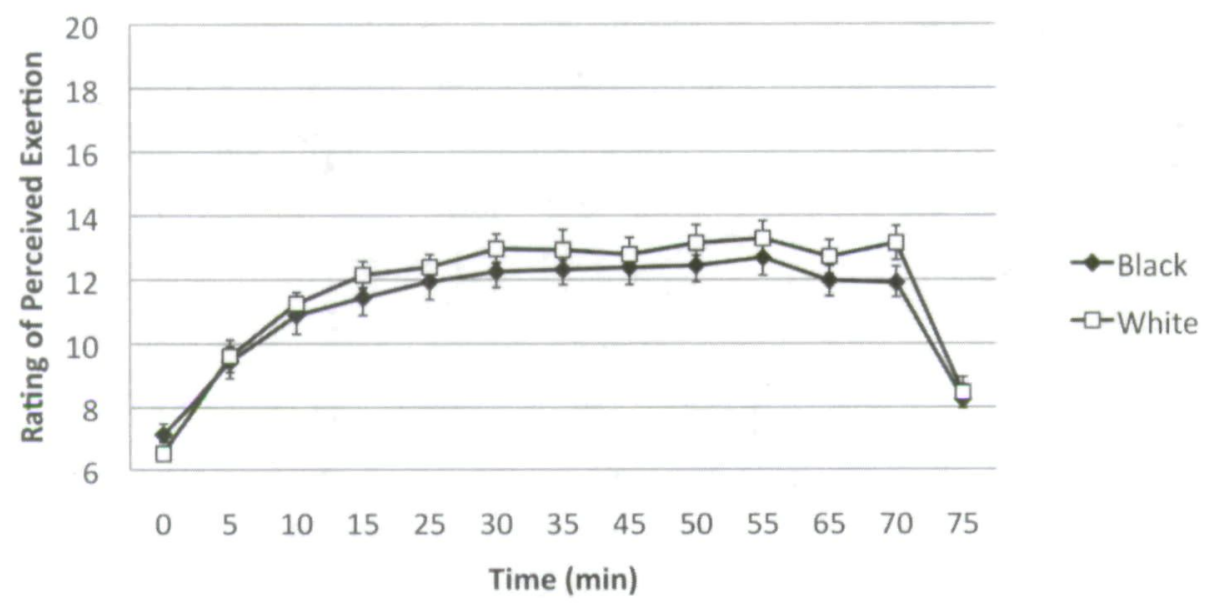

Bout 3

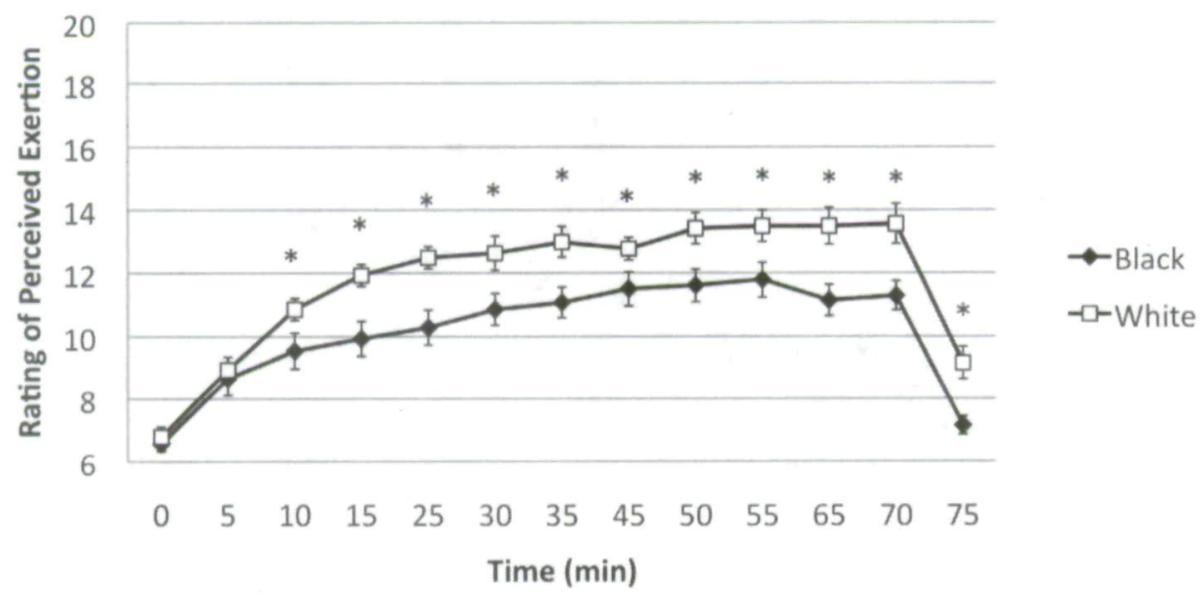

Figure $4-$ Rating of perceived exertion during exercise. Mean \pm SE. * Significant effect of exercise at $P<.05$. 
Bout 1

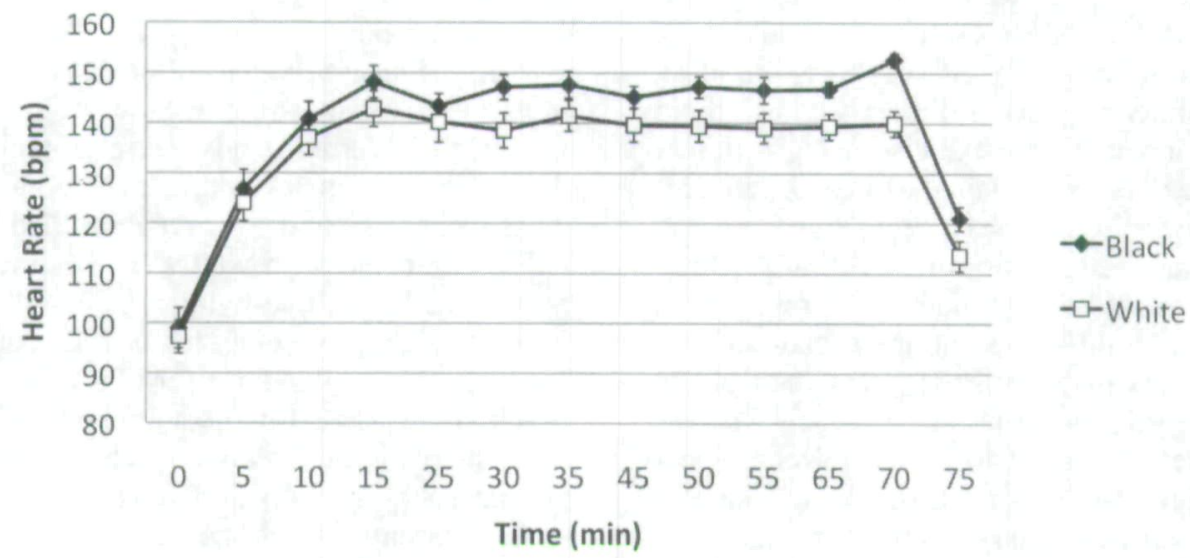

Bout 2

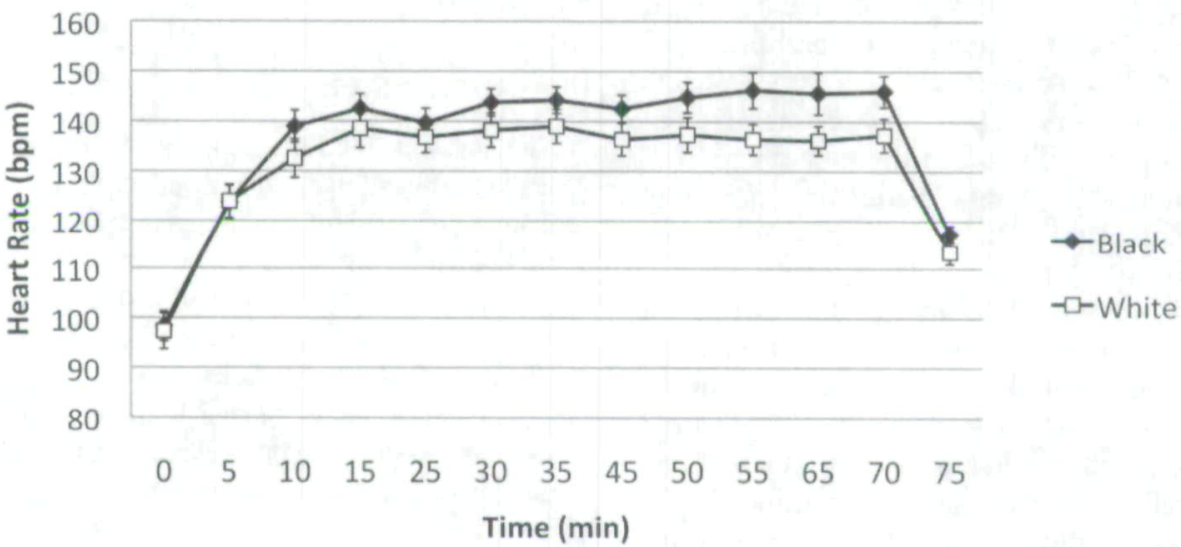

Bout 3

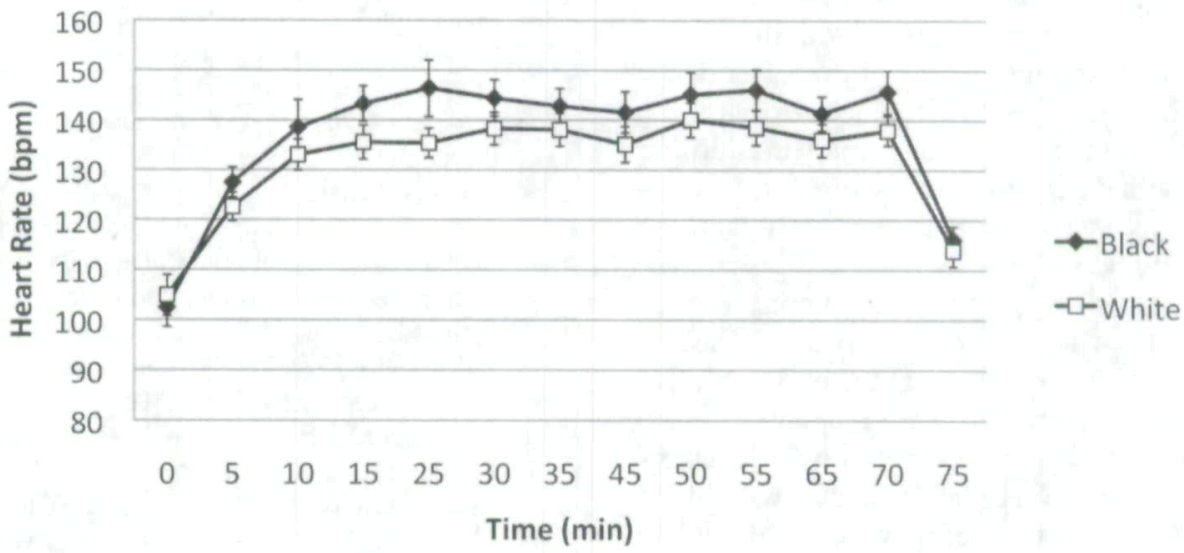

Figure 5 - Heart rate during exercise. Mean \pm SE. * Significant effect of exercise at $P<.05$. 
reported a more positive affect during exercise relative to white participants. It is possible that exercise enjoyment and/or social desirability were higher in black participants. For example, the mode of exercise (eg, walking vs. bicycling) may have been more enjoyable for the black compared with white participants. Future studies should examine in-task mood during exercise using different modes of exercise to better explain the racial differences in positive affect reported in the current study.

Timeframe and resource constraints limit the findings of the current study to be more exploratory than definitive. By design, differences in the social/environmental determinants of exercise (level of education, neighborhood safety, lack of transportation, lack of access to facilities) were minimized to isolate psychological responses to exercise in the personal domain. Thus, the use of black and white college students and faculty from the university community increased the internal validity by minimizing interindividual variability but reduced the external validity because the study environment cannot be generalized to other environments. Our findings however, provide insight regarding the independent effects of single bouts of exercise on state-anxiety, mood and task selfefficacy in black and white individuals. Future research should examine other personal and environmental constructs of $\mathrm{SCT}^{3}$ (eg, perceived health status, perceived neighborhood safety) to obtain a better understanding of their influence on exercise behavior in both black and white populations.

Several studies have demonstrated that race concordance of physician/patient in black populations is positively associated with aspects of care for adults, such as participatory decision-making, interpersonal respect, and satisfaction. ${ }^{32,33}$ In laboratory settings, there is also a fairly well-established series of findings in the psychophysiology literature that demonstrates interracial interactions promote elevations in physiology (ie, galvanic skin response) and/or negative affect in black and white populations, especially when white male participants encounter a black male researcher. ${ }^{34,35}$ Based on the above-mentioned clinical and laboratory findings, the race of the investigator was matched with the race of the black participants and the gender of the investigator was female to avoid race by sex differences in heart rate responses in the white male participants. In addition, participants met with the investigator on 3 separate occasions before completing the exercise sessions to increase trust and familiarity between participant and researcher. Despite our careful control of race and sex concordance in the current study, it is still possible that the race of the investigator could have influenced the psychological responses to exercise in the white participants. Before exercising, white participants reported higher levels of psychological distress and fatigue compared with black participants. Although it is unclear why these preexercise differences existed in our cohort, it is important to note that distress and fatigue scores were extremely low in both groups. In addition, during exercise, heart rate was similar between both racial groups and preexercise scores of distress and fatigue did not appear to influence the exercise response to mood.

In conclusion, racial differences were not present in prepost measures of self-efficacy, state-anxiety, and mood in the current study. Despite similar heart rates during exercise, blacks reported a more positive affect during exercise and lower RPE (third exercise bout only), compared with whites. If these results are supported in a larger, free-living population, improvements in psychological outcomes for exercise could have strong public health implications for blacks. Emphasizing the psychological benefits of individual bouts of exercise could increase the likelihood of black individuals completing a subsequent bout of exercise. In particular, lifestyle interventions that include messages that reinforce improvements in positive affect during exercise as well as improvements in self-efficacy and mood after exercise may prove to be effective strategies to increase LTPA as well as improve the physical and psychological health of non-Hispanic blacks.

\section{Acknowledgments}

The authors would like to thank the research participants for enthusiasm and their commitment of time and effort. We also acknowledge helpful assistance from John Staudenmayer, Ph.D., Stuart Chipkin, M.D., Brooke Stephens, Ph.D., and Steven Malin, M.S. Funding for this study was provided by American Diabetes Association 7-04-JF-10 and an American College of Sports Medicine Doctoral Research Grant. The authors do not have a financial relationship with the American Diabetes Association or the American College of Sports Medicine, whom sponsored the research.

\section{References}

1. Control CD, ed. Trends in leisure-time physical activity: a report on recommendations of the task force on community preventive services. In: MMWR. 2005;54:991-994.

2. Control CD, ed. Increasing physical activity: a report on recommendations of the task force on community preventive services. Atlanta. 2001.

3. Bandura A. Self-efficacy: the exercise of control. New York: Freeman; 1997.

4. Ainsworth BE, Wilcox S, Thompson WW, Richter DL, Henderson KA. Personal, social, and physical environmental correlates of physical activity in African-American women in South Carolina. Am J Prev Med. 2003;25:23-29.

5. Felton GM, Boyd MD, Bartoces MG, Tavakoli AS. Physical activity in young African American women. Health Care Women Int. 2002;23:905-918.

6. Voorhees CC, Rohm Young D. Personal, social, and physical environmental correlates of physical activity levels in urban Latinas. Am J Prev Med. 2003;25:61-68.

7. Wilbur J, Chandler PJ, Dancy B, Lee H. Correlates of physical activity in urban Midwestern African-American women. Am J Prev Med. 2003;25:45-52. 
8. Wilbur J, Michaels Miller A, Chandler P, McDevitt J. Determinants of physical activity and adherence to a 24-week home-based walking program in African American and Caucasian women. Res Nurs Health. 2003;26:213-224.

9. Eyler AA, Brownson RC, Donatelle RJ, et al. Physical activity social support and middle- and older-aged minority women: results from a US survey. Soc Sci Med. 1999;49:781-789.

10. Bungum TJ, Morrow JR, Jr. Differences in self-reported rationale for perceived increases in physical activity by ethnicity and gender. Res Q Exerc Sport. 2000;71:55-60.

11. Banks-Wallace J, Conn V. Interventions to promote physical activity among African American women. Public Health Nurs. 2002;19:321-335.

12. Trost SG, Owen N, Bauman AE, Sallis JF, Brown W. Correlates of adults' participation in physical activity: review and update. Med Sci Sports Exerc. 2002;34:1996-2001.

13. North TC, McCullagh P, Tran ZV. Effect of exercise on depression. Exerc Sport Sci Rev. 1990;18:379-415.

14. Salmon P. Effects of physical exercise on anxiety, depression, and sensitivity to stress: a unifying theory. Clin Psychol Rev. 2001;21:33-61.

15. Landers DM, S.J. Petruzzello: Physical activity, fitness, and anxiety. Physical activity, fitness, and health: international proceedings and consensus statement, 1994.

16. Cox RH, Thomas TR, Hinton PS, Donahue OM. Effects of acute bouts of aerobic exercise of varied intensity on subjective mood experiences in women of different age groups across time. J Sport Behav. 2006;29:40-60.

17. Arent SW, Wilcox S, Thompson WW, Richter DL, Henderson KA. Personal, social, and physical environment correlates of physical activity in African American women in South Carolina. Am J Prev Med. 2003;25:23-29.

18. King AC, Castro C, Wilcox S, et al. Personal and environmental factors associated with physical inactivity among different racial-ethnic groups of U.S. middle-aged and older- aged women. Health Psychol. 2000;19:354-364.

19. Focht BC, Knapp DJ, Gavin TP, Raedeke TD, Kickner RC. Affective and self-efficacy responses to acute aerobic exercise in sedentary older and younger adults. $J$ Aging Phys Act. 2007;15:123-138.

20. Marquez DX, Jerome GL, McAuley E, Snook EM, Canakisova S. Self-efficacy manipulation and state anxiety responses to exercise in low active women. Psychol Health. 2002;17:783-791.
21. Jerome GJ, Marquez DX, McAuley E, et al. Self-efficacy effects on feeling states in women. Int J Behav Med. 2002;9:139-154.

22. Speilberger CD, Gorsuch RL, Luschene RE. The state trait anxiety inventory manual. Palo Alto, CA: Consulting Psychologists Press; 1969.

23. McAuley E. The subjective exercise experiences scale (SEES): development and preliminary validation. J Sport Exer Psychol. 1994;16:163-177.

24. Daley AJWA. Subjective exercise experiences during and after high and low intensity exercise in active and inactive adult females. Some preliminary findings. J Sports Med Phys Fitness. 2003;43:220-222.

25. Daley A, Welch A. The effects of $15 \mathrm{~min}$ and $30 \mathrm{~min}$ of exercise on affective responses both during and after exercise. J Sports Sci. 2004;22:621-628.

26. Rudolph DL, Butki BD. Self-efficacy and affective responses to short bouts of exercise. JAppl Sport Psychol. 1998;10:268-280.

27. Borg G. Perceived exertion as an indicator of somatic stress. Scand J Rehabil Med. 1970;2:92-98.

28. Russell WD. On the current status of rated perceived exertion. Percept Mot Skills. 1997;84:799-808.

29. Crase D, Rosato FD. Exercise and aging: new perspectives and educational approaches. Educ Gerontol. 1979;4:367376.

30. Petruzello S, Landers D, Hatfield B, Kubitz K, Salazar W. A meta-analysis on the anxiety reducing effects of acute and chronic exercise. Sports Med. 1991;11:143-182.

31. Institute of Medicine of the National Academies. Dietary reference intakes: energy, carbohydrate, fiber, fatty acids, cholesterol, protein, and amino acids. Washington, D.C.: The National Academies Press; 2002.

32. Cooper-Patrick L, Gallo JJ, Gonzales JJ, et al. Race, gender, and partnership in the patient-physician relationship. JAMA. 1999;282:583-589.

33. Saha S, Komaromy M, Koepsell TD, Bindman AB. Patientphysician racial concordance and the perceived quality and use of health care. Arch Intern Med. 1999;159:997-1004.

34. Fisher LEKH. Race differences and experimenter race effect in galvanic skin response. Psychophyiology. 1973;10:578-582.

35. Vrana RD, Sr. Physiological response to a minimal social encounter: effects of gender, ethnicity, and social context. Psychophysiology. 1998;35:462-469. 
Copyright of Journal of Physical Activity \& Health is the property of Human Kinetics Publishers, Inc. and its content may not be copied or emailed to multiple sites or posted to a listserv without the copyright holder's express written permission. However, users may print, download, or email articles for individual use. 